\title{
Dinámicas metropolitanas y tiempos de la movilidad. La región metropolitana de Barcelona, como ejemplo ${ }^{1}$
}

\author{
Carme Miralles-Guasch \\ Departamento de Geografía \\ Universitat Autónoma de Barcelona \\ carme.miralles@uab.es
}

Recibido: 7 de Marzo de 2010

Enviado a evaluar: 9 de Marzo de 2010

Aceptado: 24 de Febrero de 2011

\section{RESUMEN}

Las áreas metropolitanas se han analizado desde las relaciones espaciales que desarrollan los desplazamientos por motivo trabajo. Este artículo quiere complementar esta visión unifuncional de las realidades metropolitanas, introduciendo los desplazamientos cotidianos como actividades que estructuran territorios desde todos los motivos que los inducen, tanto los laborales como los personales.

Además el tiempo de desplazamiento es una variable relacionada con los usos del tiempo que se otorgan a otras actividades, considerándolo un valor social, con sus complementariedades y sus contradicciones. Hasta el punto que la conciliación de tiempos es también una conciliación de espacios metropolitanos. Aquí las dinámicas metropolitanas, utilizando la Región Metropolitana de Barcelona como ejemplo, se analizan desde los tiempos de la movilidad y de ellos se perfilan las actividades cotidianas enlazadas con la lejanía espacial y los medios de transporte mecánicos y también las funciones cotidianas que tienen en la proximidad, en la intensificación del barrio y en los medios de transporte no mecánicos sus principales características.

Palabras clave: Región Metropolitana de Barcelona, movilidad, tiempo social, transportes

1Este artículo es una reelaboración basada en la ponencia: El territorio metropolitano y los tiempos de la movilidad. El Caso de la Región Metropolitana de Barcelona presentada en el Congreso Asociación de Geógrafos Españoles, Ciudad Real, 2009. Además, este trabajo ha sido financiado por el proyecto CSO2010-18022 (subprograma GEOG). 


\title{
Metropolitan dynamics and mobility times. Metropolitan region of Barcelona as example
}

\begin{abstract}
Metropolitan areas have been studies from the perspective of the spatial relationships mapped out by work commutes. This article attempts to complement this single-function view of reality of metropolitan life by presenting everyday journeys as activities that structure territories from the perspective of all travel-related motives, both work related and for personal reasons.

In addition, travel time is a variable which is related to the uses of time spent on other activities. It is seen as a social value with its corresponding complementary and contradictory aspects to the extent that reconciling time also means reconciling metropolitan spaces. Using the Metropolitan Region of Barcelona (MRB) as an example, this article analyses metropolitan dynamics from the perspective of mobility times and from these gives an outline of everyday activities linked to spatial distance and mechanical means of transport, and also their everyday functions in proximity spaces, in the intensification of the neighbourhood and in the non-mechanical means of transport their principal characteristics.
\end{abstract}

Key words: Metropolitan Region of Barcelona, mobility, social time, transport systems.

\section{Les dynamiques métropolitaines et temps de mobilité. La région métropolitaine de Barcelone à titre d'exemple}

\section{RÉSUMÉ}

Les régions métropolitaines ont été étudiées à partir d'une perspective basée sur les relations spatiales par rapport au déplacement au travail. Cet article tente de compléter cette vision de la réalité de la vie métropolitaine en présentant les déplacements quotidiens, qu'ils soient pour des raisons professionnelles ou personnelles, comme activités structurant le territoire.

En outre, le temps de voyage est une variable qui est reliée à l'utilisation au temps consacré aux autres activités. On le perçoit comme une valeur sociale avec ses aspects complémentaires et contradictoires dans la mesure où concilier le temps signifie aussi concilier les espaces métropolitains. En prenant comme exemple la Région métropolitaine de Barcelone (RMB), cet article analyse les dynamiques métropolitaines à partir des perspectives du temps de mobilité et donne ainsi au plan des activités quotidiennes liées à la distance spatiales et aux moyens de transport mécaniques et leur fonction quotidienne reliée aux espaces de proximité, à l'intensification du voisinage et aux moyens de transport non-mécaniques leurs principales caractéristiques.

Mots clés: Région métropolitaine de Barcelone, mobilité, temps social, systèmes de transport.

\section{INTRODUCCIÓN}

Las políticas urbanas ponen cada vez más atención en el vínculo existente entre el funcionamiento de las ciudades y el uso del tiempo de sus ciudadanos. De hecho los vectores espaciales clásicos de la ordenación del territorio (densidad, morfología, 
planeamiento...) se están articulando con el vector social del tiempo, hasta tal punto que la conciliación de tiempos es también una conciliación de territorios. La hipótesis de partida es que la estructura del territorio metropolitano y la organización de sus ciudades determinan en gran medida las estrategias de los ciudadanos en el desarrollo de sus actividades cotidianas, y con ellas sus desplazamientos y el tiempo invertido en ellos. Por lo que un análisis de los tiempos cotidianos permite explorar las nuevas dinámicas metropolitanas.

La reflexión quiere avanzar en los territorios que, -como las áreas metropolitanas, como un sistema de territorios físicos y de estructuras sociales-, tienen una doble relación con el tiempo. Por una parte con el tiempo largo, que modela los paisajes, la memoria colectiva y la construcción identitaria. Por el otro el tiempo cotidiano, el de los desplazamientos de la proximidad o de la lejanía, y del territorio contiguo o diseminado.

Todo ello se explora en la Región Metropolitana de Barcelona, donde se identifican las dinámicas urbanas y los modelos de movilidad que la definen, para poder focalizar el análisis en la vida cotidiana de la proximidad o de la distancia, con relación a los tiempos de la movilidad. Este objetivo se aborda con una exposición teórica sobre los tiempos sociales en la ciudad, como una coordenada que junto a la coordenada espacial, dibuja los espacios cotidianos en las regiones metropolitanas. Después el artículo define el modelo urbano en la Región Metropolitana de Barcelona a partir de los datos de la población por ámbitos submetropolitanos, en relación a las distintas densidades poblacionales, y a los cambios de ubicación de las actividades económicas en estos territorios. Para completar el análisis se define el modelo de movilidad de los habitantes de este espacio metropolitano, a partir de los desplazamientos medios realizados y de la distribución modal que estos tienen en relación a los distintos territorios submetropolitanos y a los motivos que los generan. La tercera parte del trabajo analiza la movilidad en relación a los tiempos de los desplazamientos y a los destinos de estos para ahondar en la relación entre las dinámicas metropolitanas, como espacios urbanos que incrementan las relaciones de lejanía, pero que a la vez mantienen la proximidad territorial como un valor que identifica a las ciudades de este entorno.

Las fuentes de información utilizadas han sido las que ofrecen las encuestas que de forma específica y regular que desarrollan en el área de estudio. Por una parte, la Encuesta de Movilidad Cotidiana que se realizó en el año 2006 (EMQ06) en la Comunidad Autónoma Catalana. Esta operación estadística, sólo para la Región Metropolitana de Barcelona se inicio en el año 1996, con una frecuencia quinquenal. También se ha utilizado la Encuesta de Condiciones de Vida y Hábitos de la población que se realiza desde 1986, cada cinco años, en el entorno Barcelonés. Esta encuesta, aunque recoge información general sobre las características de la población, tiene varios epígrafes dedicados a los lugares donde la población realiza las actividades cotidianas, según su zona de residencia en los distintos ámbitos submetropolitanos Una información que permite indagar, a partir de las actividades realizadas cerca o lejos de los lugares donde se reside, las dinámicas metropolitanas de la proximidad o de la lejanía. 


\section{LOS TIEMPOS DE LA CIUDAD}

Las áreas metropolitanas, unidades territoriales complejas, se pueden definir como la suma de territorios físicos y de estructuras sociales. Este dialogo entre realidad espacial y estructura social implica que la cotidianidad dependa, entre otros elementos del lugar donde se viva. En palabras de Davouidi (2009): la calidad de los lugares puede influir en la calidad de la vida cotidiana. Por lo que los modelos sociales no sólo incluyen interdependencias sociales sino también territoriales. Y una de las variables que interrelaciona estas dos dimensiones es el tiempo social, una medida temporal que relaciona actividades y lugares (May and Thrift, 2001).

Las sociedades y los territorios metropolitanos están caracterizadas por un doble proceso que intensifica su complejidad, uno a nivel colectivo y otro individual. Las estructuras sociales incrementan su complejidad con diversidades e interdependencias cada vez más numerosas debidas, en parte, a la profundización de la división del trabajo, a la diferenciación de los espacios y a la variedad de estratificaciones sociales, a la multiplicidad de culturas, etc. Pero también y tal como menciona Bailly (2005) las sociedades actuales se definen, cada vez más, por la flexibilidad y la individualización, por un el aumento de la disparidad de modelos de vida y evolución de las estructuras familiares. Uno y otro proceso transforman los ritmos de la vida cotidiana y revalorizan las prácticas de la movilidad y de la gestión de la ciudad (Bonfiglioli, 2009). A la vez la forma como se estructura el territorio metropolitano y como se organizan sus ciudades determina en gran medida las estrategias personales en el desarrollo de sus actividades cotidianas. De lo que deriva que al incrementarse las interdependencias aumentan también los grados de libertad individual (Tabboni, 2006).

Dentro de estas formas de vida metropolitanas destaca, por su incidencia sobre las dinámicas territoriales, la gestión cotidiana de los distintos usos sociales del tiempo. Y aunque el territorio siempre ha tenido que ver con el tiempo histórico, aquel que modela los paisajes y la memoria colectiva, -que, según Giddens (1984), tiene tres dimensiones temporales y según Parkes y Thirft (1980) sólo dos, una a corto y otra a largo plazo- en el artículo el tiempo es una variable social que parte de las actividades de los ciudadanos de estos territorios urbanos o metropolitanos a lo largo de distintas unidades temporales, sean días, semanas o años. Lo que interesa aquí no es tanto la cuantificación del tiempo en si, como las distintas actividades que se realizan a lo largo de los diversos tiempos y entrever las complementariedades y los conflictos que estas tienen (Crang, 2001).

Esta nueva dimensión de la variable temporal se hizo presente en la medida que el tiempo de trabajo mercantil perdía protagonismo a favor de otras actividades lúdicas o de ocio y también en la medida que los estudios de género reclamaban el valor de los trabajos no renumerados y hacían emerger el concepto de las dobles jornadas laborales (Prats, 1998; Torns, 2007). Así el tiempo social mide las actividades de la población a lo largo de la jornada o de la semana y en ellas distingue los tiempos dedicados al trabajo remunerado, al no remunerado, al ocio y al descanso, y a partir de ello se valoran las cargas de trabajo que tiene los distintos colectivos según la estructura social y territorial. 
El tiempo de la movilidad, como parte de este tiempo social, se caracteriza por una coordenada espacial y otra temporal. La primera se aproxima a los usos de la ciudad, relacionados con las localizaciones de las actividades en el territorio y con la capacidad o posibilidad de acceso a estas que tiene la ciudadanía. La coordenada temporal, complementaria a la anterior, define la utilización del tiempo respecto al conjunto de las actividades cotidianas, entre las que se incluye el desplazamiento. La primera coordenada se conoce como el uso de la ciudad y la segunda por los usos sociales del tiempo (Robert, 1992; Miralles, 2006).

El uso social del territorio influye en el tiempo cotidiano. Los lugares donde se trabaja, se compra, se vive, dibujan un conjunto de interrelaciones y de conexiones que se gestionan a través de la planificación y la ordenación del territorio, así como de la construcción y la gestión de las redes de transporte. La configuración física del espacio y de las infraestructuras de soporte a la movilidad condicionan las dinámicas territoriales, que a la vez determinan los tiempos de desplazamiento (Mückenberg, 2009).

El tiempo de la movilidad tiene que valorarse desde esta perspectiva global de los tiempos y desde la realidad cotidiana de sus ciudadanos, integrada dentro del conjunto de actividades que consumen tiempo. El tiempo del desplazamiento no es un valor cuantitativo y aislado, sino que es necesario interpretarlo en relación a las otras esferas del tiempo. Desde la perspectiva del ciudadano, las diversas actividades y el tiempo que les dedican no están aislados. Al contrario el tiempo es un valor único y continuo. Es el tiempo de la vida cotidiana, donde confluyen (en competencia o complementariedad) las distintas actividades. La compatibilidad de las diversas tareas se explica según el ciclo vital de las personas, definido por la edad, la actividad profesional y la relación con el espacio doméstico. Desde un punto de vista económico, de Rus (2006) afirma que a menudo el ahorro de tiempo es la principal fuente de beneficios sociales de una nueva infraestructura.

Los elementos que definen las dinámicas urbanas y metropolitanas, -como son las políticas territoriales y urbanísticas, el mercado inmobiliario y el coste de la vivienda, las infraestructuras de transporte, la localización de los lugares de trabajo y las actividad económicas, la ubicación de los servicios básicos y de los equipamientos y la evolución sociodemográfica y las migraciones poblacionalesson factores decisivos en la organización de los tiempos sociales y también de los tiempos de desplazamiento.

Las ciudades, y con ellas las áreas metropolitanas, están inmersas en dos dinámicas territoriales contrapuestas y a la vez complementarias. Por una parte se esta asistiendo a una ampliación de la ciudad real, a través de la descentralización territorial y de la especialización funcional del entono urbano, lo que genera una mayor apertura e interrelación de los municipios y por lo tanto una ampliación en términos físicos de la ciudad cotidiana. Dinámicas territoriales que han influenciado la creación e intensificación de las áreas metropolitanas y que han entrañado un aumento de las distancias y del tiempo de desplazamiento. La interrelación de la ciudad en su entorno más inmediato se intensifica. Dispersión, integración y especialización son dinámicas que caracterizan la realidad urbana, y más intensamente aquella que ya es metropolitana, y determinan los usos sociales del territorio. 
Uno de los fenómenos que más influyen en esta dispersión y en la consecuente ampliación de la ciudad real, del espacio donde los ciudadanos desarrollan su actividad cotidiana, son los lugares de trabajo en relación a los de residencia. En muchas ocasiones se ha leído el territorio desde esta actividad cotidiana, relacionando los territorios laborales y de residencia. En estos términos se definen los mercados de trabajo (Riera, 1989; Castañer et al., 2001; Capel 2005; Feria y Susino, 2005, Salom y Casado, 2007, Roquer, 2007).

Sin embargo, aquí se trata de incluir los desplazamientos que generan las distintas actividades cotidianas -laborales, comerciales, lúdicas, etc. - y relacionar los territorios que entre ellas se relacionan y en consecuencia sus tiempos de recorrido. De hecho, se trata, tal como apunta Torns (2007) en términos sociológicos, de interpretar el territorio metropolitano incluyendo los destinos de las actividades que dibujan la cotidianidad en su conjunto y no sólo el trabajo.

A partir de estas múltiples inclusiones mencionadas, en ciertas ciudades, se observa también un fenómeno paralelo e inverso, como es la intensificación del barrio, un hecho asociado a la búsqueda de la proximidad como un valor en alza (Serra, 2007). Una proximidad que se expresa en los destinos relacionados con las compras, con los servicios y equipamientos que se utilizan o en las actividades lúdicas o culturales. Es decir en aquellos destinos donde el usuario tiene cierta capacidad en decidir donde realiza sus actividades. Un fenómeno que parece contradictorio respecto al que se ha hecho referencia en términos ocupacionales, pero que, analizado desde una perspectiva temporal se revela complementario.

Estas dinámicas territoriales son elementos fundamentales en la gestión del tiempo, hasta el punto que la conciliación territorial es también una conciliación temporal. Además todas ellas se basan en unas redes de transporte urbano y metropolitano, consecuencia de unas políticas públicas y privadas que pueden acortar o ampliar el tiempo de desplazamiento.

Así en el ámbito concreto de la movilidad, de los desplazamientos cotidianos, estos recientes análisis han constatado un doble comportamiento que, ampliando el significado del término, se podría cualificar de bipolar, desde el punto de vista del uso del territorio, pero también complementario, desde una perspectiva del uso social del tiempo, tanto a nivel colectivo como individual. Por una parte, las tendencias detectadas han generado una mayor apertura e interrelación de los municipios, traducido en la ampliación de la ciudad real, en un aumento de las distancias y de paso en un incremento del tiempo de los desplazamientos. Por otra, una intensificación de las actividades en el mismo barrio, asociada a la búsqueda de proximidad como un valor en auge en una sociedad en que el tiempo es cada vez más un bien escaso y limitado, y donde su gestión y control es percibido como un elemento ligado a la calidad de vida (Durán, 2007). 


\section{EL MODELO URBANO Y DE MOVILIDAD EN LA REGIÓN METROPO- LITANA DE BARCELONA}

Las dinámicas territoriales antes mencionadas, caracterizadas por movimientos de concentración y dispersión que definen la Región Metropolitana de Barcelona (RMB) tienden a localizarse de forma desigual. El primer fenómeno se da en la parte central de la RMB, -en la ciudad de Barcelona y su continuo urbano- y en las partes centrales de las ciudades medias de su entorno. Unos lugares caracterizados por la compacidad y la complejidad del tejido urbano. La dispersión se ubica en las periféricas, y se basa en las bajas densidades y la separación de las funciones y las actividades. Las variables utilizadas para definir estos dos modelos, y con ellos el tiempo de la ciudad, son la distribución de la población, las tipologías urbanas y la localización de las actividades económicas en el territorio metropolitano.

Estas dinámicas metropolitanas influyen en los modelos de movilidad, los cuales se definen según los medios de transporte utilizados, los motivos y las relaciones de todo ello con las características de la población.

\subsection{LOCALIZACIÓN Y DISTRIBUCIÓN DE LA POBLACIÓN EN LA REGIÓN METROPOLITANA DE BARCELONA (RMB)}

Tal como indica en la figura 1 , si se considera la distribución de la población dentro de la RMB, se pueden definir tres ámbitos territoriales, equilibrados en población pero con características poblacionales y socioeconómicas distintas. En primer lugar el municipio de Barcelona, con un población de 1.615 .908 (33\%) y unas densidades cercanas al 16.000 habitantes $/ \mathrm{km} 2$. Después, la primera corona metropolitana o aglomeración central, que comprende la estructura urbana compacta alrededor de Barcelona, Su población es de 1.570.553 habitantes con una densidad de unos 2.900 habitantes $/ \mathrm{km}^{2}$. La parte más externa de la región o la segunda corona metropolitana, integrada por ciudades medianas, pequeñas y zonas rurales, con una población de 1.742.391 habitantes y una densidad media unos 668 habitantes $/ \mathrm{km}^{2}$.

Figura 1. Población por ámbitos de la Región Metropolitana de Barcelona

\begin{tabular}{|c|c|c|c|c|}
\hline Ámbito & \multicolumn{2}{|c|}{ Población 2008} & \multirow{2}{*}{ Superficie $(\mathrm{km} 2)$} & \multirow{2}{*}{ Densidad (hab./km2) } \\
\hline & Habitantes & $\%$ & & \\
\hline Barcelona & 1.615 .908 & $33 \%$ & 101,4 & $15.943,80$ \\
\hline Primera corona & 1.570 .553 & $32 \%$ & 534,6 & $2.937,81$ \\
\hline Segunda corona & 1.742 .391 & $35 \%$ & $2.605,54$ & 668,73 \\
\hline $\begin{array}{l}\text { Total región Metropolitana de } \\
\text { Barcelona }\end{array}$ & 4.928 .852 & $100 \%$ & $3.241,54$ & $1.520,53$ \\
\hline
\end{tabular}

Fuente: Elaboración propia a partir de IDESCAT y web Área Metropolitana de Barcelona

Estas diferencias en la densidad entre Barcelona y sus coronas metropolitanas reflejan dos formas distintas de urbanización y de ocupación del territorio. Por una parte Barcelona y su continuo urbano se presentan como una ciudad compacta en su forma y compleja en sus funciones. Su compacticidad deriva de un urbanismo que ha apostado por una ciudad densa y continua de los espacios urbanos, 
que se traduce en una trama de calles y plazas altamente utilizadas. A todo ello se le suma una distribución de las funciones urbanas bastante homogénea y mixta, con lo que a lo largo de toda la ciudad no existen espacios destinados a una sola función, sea esta comercial, residencial o industrial. Todas ellas características del espacio funcional central de la propia metrópolis. Por el contrario el modelo de ocupación de la periferia metropolitana, más en la segunda corona que en la primera y exceptuando los núcleos históricos de sus ciudades, se caracteriza por las bajas densidades, las discontinuidades de los tejidos urbanos y la zonificación funcional (Muñoz, 2006).

Además, la distribución de la población ha seguido una tendencia hacia la dispersión en el territorio, desde las ciudades más pobladas y de mayor densidad hacia núcleos pequeños y zonas de baja densidad (figura 2). Des de los años 80 en Barcelona y en otras ciudades medias del entorno metropolitano se han producido pérdidas de población debidas a migraciones residenciales (Pujadas, 2009). El resultado ha sido la pérdida del peso demográfico de Barcelona en relación al resto del territorio de la RMB, que ha pasado de ser el $41 \%$ en 1991 al 33\% en 2008. De todas formas la ciudad gana población en el último quinquenio, cambiando la dinámica poblacional que se observaba desde 1981, aunque este incremento no significa que se haya detenido el flujo de salida de la población hacia zonas periféricas de la RMB. Sólo en 2005 se produjo un saldo negativo de 11.500 personas por movimientos migratorios con el resto de la RMB.

En las coronas metropolitanas y especialmente en la segunda de ellas, en la parte más periférica, han experimentado un proceso inverso con el aumento de población por saldos migratorios positivos. Entre 1981 y 2008 la población de la segunda corona metropolitana pasó del poco más de un millón de personas a más de $1.700 .000,10$ que significa un aumento del $52 \%$ en este periodo. El crecimiento de la primera corona ha sido mucho más discreto, con un aumento de la población de apenas 180.000 personas, lo que significa un incremento del $14 \%$.

Figura 2. Evolución de la población en los distintos ámbitos de la Región Metropolitana de Barcelona

\begin{tabular}{|c|c|c|c|c|c|c|c|c|c|c|}
\hline & \multicolumn{2}{|c|}{ Población 1981} & \multicolumn{2}{|c|}{ Población 1996} & \multicolumn{2}{|c|}{ Población 2001} & \multicolumn{2}{|c|}{ Población 2005} & \multicolumn{2}{|c|}{ Población 2008} \\
\hline & Habitantes & $\%$ & Habitantes & $\%$ & Habitantes & $\%$ & Habitantes & $\%$ & Habitantes & $\%$ \\
\hline Barcelona & 1.752 .627 & $41 \%$ & 1.508 .805 & $36 \%$ & 1.503 .884 & $34 \%$ & 1.593 .075 & $33 \%$ & 1.615 .908 & $33 \%$ \\
\hline $\begin{array}{l}\text { Primera } \\
\text { corona }\end{array}$ & 1.392 .386 & $33 \%$ & 1.410 .805 & $33 \%$ & 1.432 .697 & $33 \%$ & 1.542 .683 & $32 \%$ & 1.570 .553 & $32 \%$ \\
\hline $\begin{array}{l}\text { Segunda } \\
\text { corona }\end{array}$ & 1.093 .863 & $26 \%$ & 1.308 .438 & $31 \%$ & 1.453 .827 & $33 \%$ & 1.634 .422 & $34 \%$ & 1.742 .391 & $35 \%$ \\
\hline $\begin{array}{l}\text { Total } \\
\text { RMB }\end{array}$ & 4.238 .876 & $100 \%$ & 4.228 .048 & $100 \%$ & 4.390 .408 & $100 \%$ & 4.770 .180 & $\begin{array}{c}100 \\
\%\end{array}$ & 4.928 .852 & $100 \%$ \\
\hline
\end{tabular}

Fuente: Área Metropolitana de Barcelona

Todo ello en paralelo a la importancia creciente de los tejidos residenciales de baja densidad, los cuales no pueden ser tratados como un fenómeno minoritario en la RMB, cuando grandes zonas del territorio se caracterizan por esta tipología de vivienda. En el año 2000 se contabilizaban $6.089,2$ ha. de suelo urbano resi- 
dencial extensivo y de baja densidad, lo que representa un $40 \%$ del total del suelo residencial (MMAM, 2003). Actualmente, la producción de vivienda esta generando unas tipologías de residencias que incrementaran la presencia en la RMB de estos tejidos. Así, entre 1987 y 2001 se construyeron en la RMB 330.511 viviendas, de las que más de 205.000 , es decir el 65\%, correspondían a tipologías de baja densidad (viviendas aisladas o adosadas) (Muñoz, 2006). El mayor número absoluto de viviendas de este tipo, en concreto 206.878 , se construyeron en la segunda corona metropolitana.

\subsection{REUBICACIÓN Y DIFUSIÓN DE LAS ACTIVIDADES ECONÓMICAS EN EL TERRITORIO METROPOLITANO}

En relación a la distribución y evolución de las actividades económicas, en los últimos años, se desprenden unas conclusiones similares a las de la población. Y aunque Barcelona y los municipios del núcleo central metropolitano no dejan de ser un gran foco de actividad, como le corresponde a su papel funcional central de la metrópoli, existe un proceso de desplazamiento de las actividades económicas hacia zonas externas de los municipios, buscando mejores condiciones y accesibilidades. Esta tendencia empieza a desarrollarse con las actividades productivas y más recientemente se le suman a ella el terciario, el comercio e incluso los centros educativos y sanitarios.

La distribución y evolución de los afiliados en la seguridad social da cuenta de este proceso de descentralización de la actividad económica. Entre 1996 y 2008 en el conjunto de la RMB, el número de afiliados se incrementó el 31\%, como se puede ver en la figura 3. Crecimiento que ha sido desigual según los ámbitos. En Barcelona el aumento ha sido del $27 \%$, sin embargo es en la segunda corona metropolitana donde este incremento es mayor, llegando al 39\%.

Figura 3. Distribución de los puestos de trabajo en la Región Metropolitana de Barcelona

\begin{tabular}{|l|c|c|c|c|c|c|}
\hline & \multicolumn{3}{|c|}{ Puestos de trabajo } & \multicolumn{3}{c|}{ Incremento } \\
\hline & 1996 & 2002 & 2008 & $1996-2002$ & $2002-2008$ & $1996-2008$ \\
\hline Barcelona & 787.507 & 956.521 & 1.078 .820 & $18 \%$ & $11 \%$ & $27 \%$ \\
\hline Primera corona & 387.441 & 503.486 & 563.264 & $23 \%$ & $11 \%$ & $31 \%$ \\
\hline Segunda corona & 422.897 & 572.788 & 689.970 & $26 \%$ & $17 \%$ & $39 \%$ \\
\hline Total RMB & 1.599 .841 & 2.034 .797 & 2.332 .054 & $21 \%$ & $13 \%$ & $31 \%$ \\
\hline
\end{tabular}

Fuente: Mancomunitat de Municipis del Área Metropolitana de Barcelona, 2006 i Ajuntament de Barcelona; http://www.bcn.es/publicacions/b_economia/be68.pdf

El proceso de difusión de las actividades por el territorio se ha producido en paralelo a la proliferación de polígonos industriales, ubicados en zonas externas de la ciudad y aislados de las partes urbanas consolidadas (Miralles-Guasch y Donat, 2007). Además, estos polígonos se sitúan lejos de las zonas residenciales, sin que los servicios públicos de transporte, urbanos o interurbanos, lleguen hasta ellos. Aunque existen pocos datos de la situación de estos servicios de transporte público en estos espacios suburbanos en 2003 (PIRMB, 2003) se realizó un estudio sobre accesibilidad en transporte público a los polígonos industriales, a partir de la distancia que existe entre la 
parada más próxima, tanto en transporte ferroviario como en viario, y el centro del área industrial. Aunque este análisis sólo era una primera aproximación topográfica entre dos puntos, la parada y el centro del polígono, y no se pueda deducir a partir sólo de estas cifras cual es la accesibilidad real de las personas que tienen que llegar hasta ellos, si que se puede observar unas primeros y preocupante déficits. Tal y como se observa en la figura 4 buena parte de los casi 700 polígonos analizados tienen una distancia media hasta la parada más próxima alrededor de 1000 metros, lo que significa que obligan a los trabajadores a realizar un trayecto de más de 15 minutos andando. Además las características territoriales de los polígonos, como su ubicación, dispersión y atomización, como a los horarios de trabajo, (turnos de noche y de día, etc.)., hacen que sea muy difícil que los transportes colectivos, en estas condiciones, puedan ser instrumentos útiles de transporte para las personas que trabajen en ellos. (Cebollada, 2006).

Figura 4. Distancia del centro de los polígonos industriales de la Región Metropolitana de Barcelona a una parada de transporte público más cercana

\begin{tabular}{|l|c|c|c|c|}
\hline Distancia a parada de TPC & Número de polígonos & $\%$ & Superficie industrial & $\%$ \\
\hline menos $750 \mathrm{~m}$ & 401 & 58 & 8.597 & 54 \\
\hline $750-1.500 \mathrm{~m}$ & 159 & 23 & 4.156 & 26 \\
\hline Más de $1.500 \mathrm{~m}$ & 134 & 19 & 3.173 & 20 \\
\hline Total & 694 & 100 & 15.926 & 100 \\
\hline
\end{tabular}

Fuente: Pacte Industrial de la Región Metropolitana de Barcelona (PIRMB)

\subsection{EL MODELO DE MOVILIDAD EN LA REGIÓN METROPOLITANA DE BARCELONA}

Los desplazamientos están directamente relacionados con los sistemas de interrelación social y con la distribución de las actividades económicas sobre el territorio (Camagni et al., 2002). Por lo que los modelos de movilidad (caracterizados por los tiempos, los motivos y los medios de transporte) derivan de variables que dependen de las distribuciones espaciales de las actividades y de los tipos de urbanización. Los modelos de movilidad son también modelos territoriales (Wiel, 1999), por lo que la transformación de las estructuras económicas y territoriales alteran los desplazamientos cotidianos de las personas.

De los datos obtenidos anualmente por las Encuestas de Movilidad en la RMB se concluye, tal como indica la Figura 5, un aumento del número de desplazamientos entre el 2004 y el 2006 de un $20 \%$ y un estancamiento en el año 2008, con lo que estos alcanzan los 14 millones de movimientos diarios. Con un ratio que oscila entre $\operatorname{los} 3,47$ y $\operatorname{los} 3,64$ desplazamientos por persona y día.

Figura 5. Desplazamientos en la Región Metropolitana de Barcelona 2004-2008

\begin{tabular}{|l|c|c|c|}
\hline & 2004 & 2006 & 2008 \\
\hline Desplazamientos diarios & 13.024 .206 & 15.801 .351 & 14.250 .255 \\
\hline Media de desplazamientos día/persona* & 3,47 & 3,64 & 3,63 \\
\hline
\end{tabular}

Fuente. ATM y Generalitat Catalunya, 2004, 2006, 2008 
También se observa un aumento de la longitud de desplazamientos, para ello es necesario recurrir a los datos de la $\mathrm{EMO}^{2}$ que muestran que durante el período de veinte años que va des de 1981 hasta el 2001, la distancia media de los desplazamientos por motivo estudios y trabajo ha pasado de 9,6 a $12,9 \mathrm{~km}$.

Por lo que se refiere a los modos de transporte utilizados, de los 101, 2 millones de desplazamientos semanales hechos por los residentes de la Región Metropolitana de Barcelona, 45,8 millones $(45,2 \%)$ son en modos no motorizados, 38,2 millones $(37,8 \%)$ con transporte privado y el 17,2 millones $(17,0 \%)$ con transporte público (figura 6). En día laborable el uso del transporte público es más elevado que en los sábados y los festivos (el 18,6\% y $11,1 \%$, respectivamente, tendencia que se invierte en el uso del transporte privado (35,8\% y $44,7 \%$, respectivamente).

Figura 6. Distribución modal de los desplazamientos en la Región Metropolitana de Barcelona. 2006

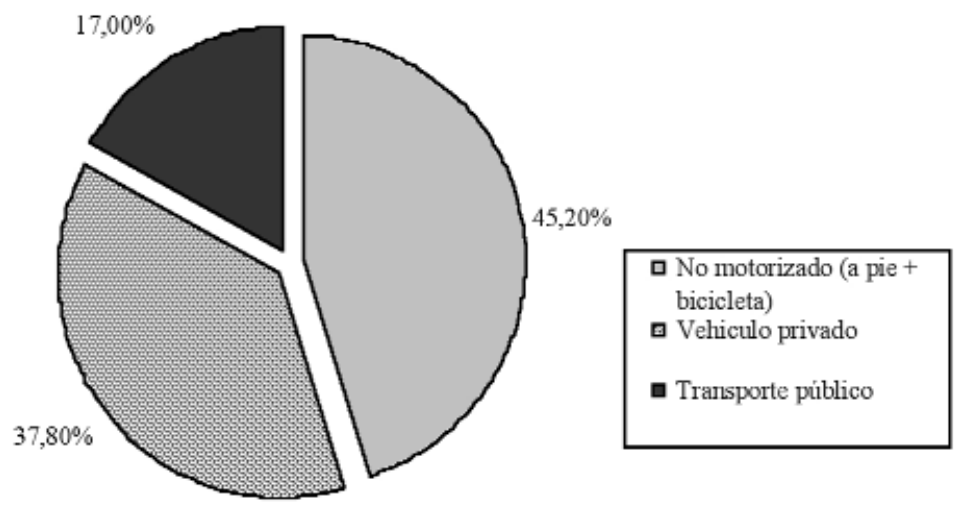

Fuente: ATM y Generalitat Catalunya, 2006

Casi la totalidad de los desplazamientos con modos no motorizados corresponden a los movimientos a pie, de los que un 30\% están por debajo de los 5 minutos. La utilización de la bicicleta significa sólo un 1,6\% en día laborable y el $2 \%$ en día festivo. En transporte público, los modos más urbanos como el metro y el autobús urbano son los más usados, con el $33,4 \%$ y el $28,1 \%$ del total de recorridos hechos en este medio de transporte en días laborables. En el transporte privado, el coche es el más utilizado con una cuota del $86,9 \%$ en día laborable y el $94,0 \%$ en fin de semana. También existe un incremento de la ocupación media por vehiculo que pasa del 1,29 personas por coche en laborable a 1,74 en festivo.

A nivel territorial se puede hablar de dos modelos de movilidad distintos, según los usos de los diversos modos de transporte. La mayor dotación de transporte públi-

2 Hasta 2001 se realizaba la Encuesta de Movilidad Obligada, que a pesar de su nombre no consistía en una encuesta sino en la recopilación de información a partir del censo. Permitía un estudio exhaustivo de la población y con una gran desagregación espacial, aunque sólo recogía información sobre el primer desplazamiento por motivo de trabajo o estudio. 
co en Barcelona (y por extensión a su continuo urbano) determina que este sea el ámbito con mayores proporciones de uso de este transporte, con cuotas de alrededor del $30 \%$ en día laborable y del $20 \%$ en día festivo. Este hecho junto a un porcentaje mayor de desplazamientos no motorizados, que significan casi la mitad de todos los movimientos, influye que, en esta área central de la RMB, el uso del transporte privado sea menor. Una dinámica opuesta aparece en la medida que nos alejamos del centro de la metrópolis, donde el peso del transporte privado se incrementa en menoscabo del uso del transporte público (Figura 7).

Figura 7. Medios de transporte en la Región Metropolitana de Barcelona. 2006

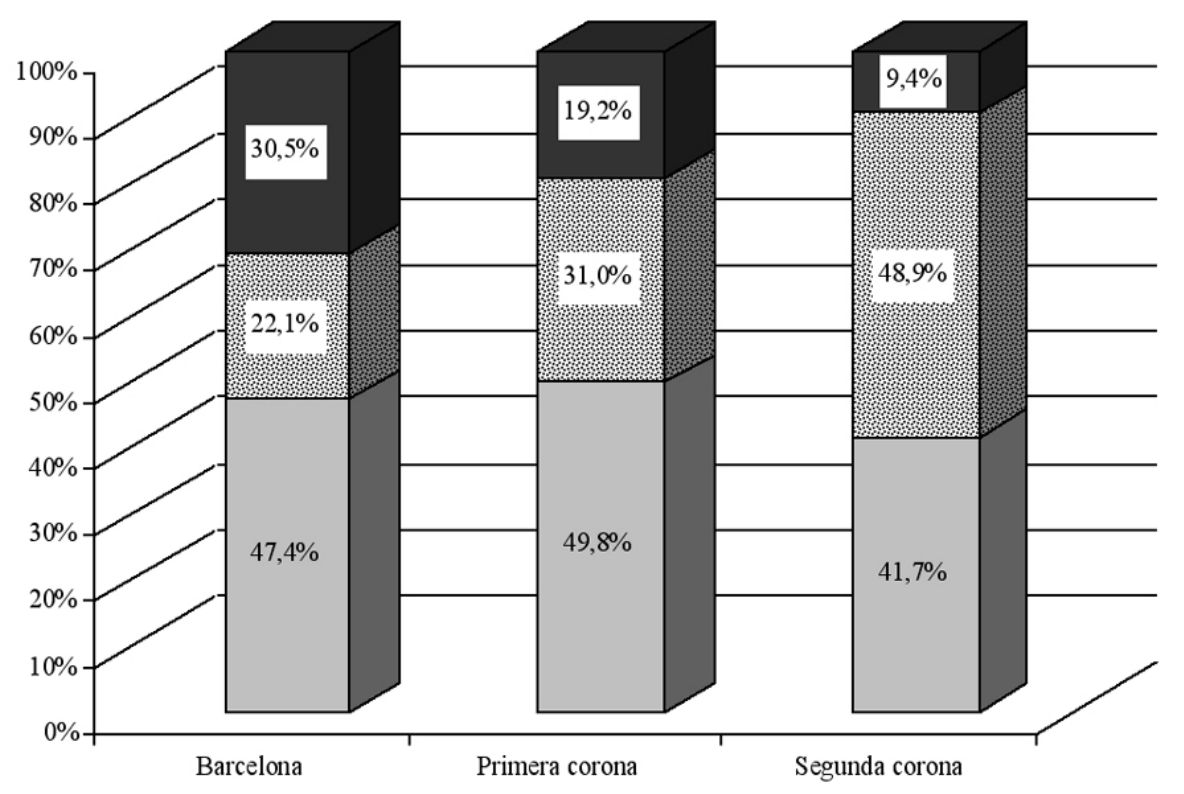

$\square$ No motorizado (a pie + bicicleta) $\quad$ a Vehiculo privado $\square$ Transporte público

Fuente: ATM y Generalitat de Catalunya , 2006

Por lo que se refiere a los motivos ${ }^{3}$ de desplazamiento (Figura 8) de los residentes en la Región Metropolitana de Barcelona, alrededor del 34\% son por motivo personal, un $20 \%$ ocupacional y el $45,5 \%$ se concentran en los retornos al lugar

3 La Encuesta de movilidad Cotidiana 2006 diferencia tres grupos de motivos: el ocupacional, el personal y los regresos al lugar de residencia. En los primeros se agrupan los motivos laborales y de estudios. Entre los personales se encuentran las compras, el ocio, las visitas, los paseos, etc. En total en la Encuesta se hallan 14 motivos distintos que originan los desplazamientos. 
de residencia. El motivo de desplazamiento es distinto según la tipología de día de la semana. Las principales diferencias se producen en los motivos ocupacionales, que en los fines de semana se reducen al 3,7\% del total, en beneficio de la movilidad personal que llega a ocupar hasta el $50 \%$ de la cuota. A nivel territorial destaca el peso que los motivos personales tienen en la ciudad de Barcelona, tanto en laborable como en festivo. La estructura de edades de la población puede explicar esta diferencia territorial.

Figura 8. Distribución de desplazamientos según motivo. 2006

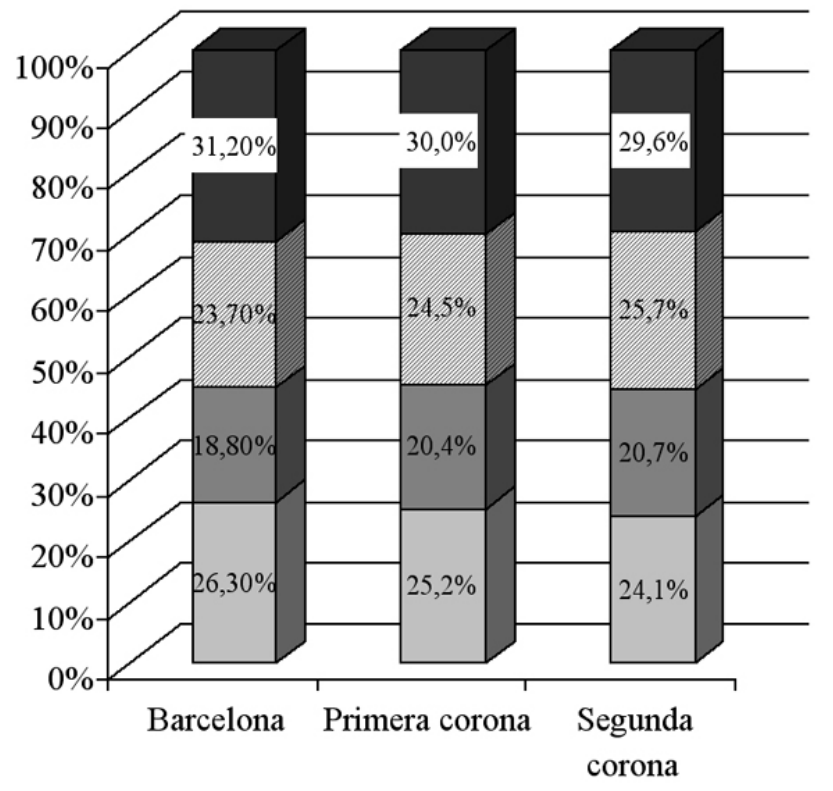

$\square$ Movilidad personal

\section{Movilidad ocupacional}

$\square$ Retorno a residencia por motivo personal

$\square$ Retorno a residencia por motivo ocupacional

Fuente: ATM y Generalitat de Catalunya, 2006

Puede señalarse, también, una correlación entre los modos de transporte y los motivos. El $46,8 \%$ de la movilidad por trabajo y estudio se realiza en vehículo privado. Este predominio deriva del proceso de dispersión de la población y las actividades - descrito en el apartado anterior- que se ha producido en los últimos años. Una dispersión que obliga a recorrer grandes distancias para acceder al puesto de trabajo $\mathrm{y}$ en las que el vehiculo privado resulta imprescindible. Por el contrario, la movilidad a pie predomina cuando los motivos del desplazamiento son personales (compras, ocio, visitas...), con un porcentaje de casi el 60\% (Figura 9). 
Figura 9. Motivos y medios de transporte. 2006

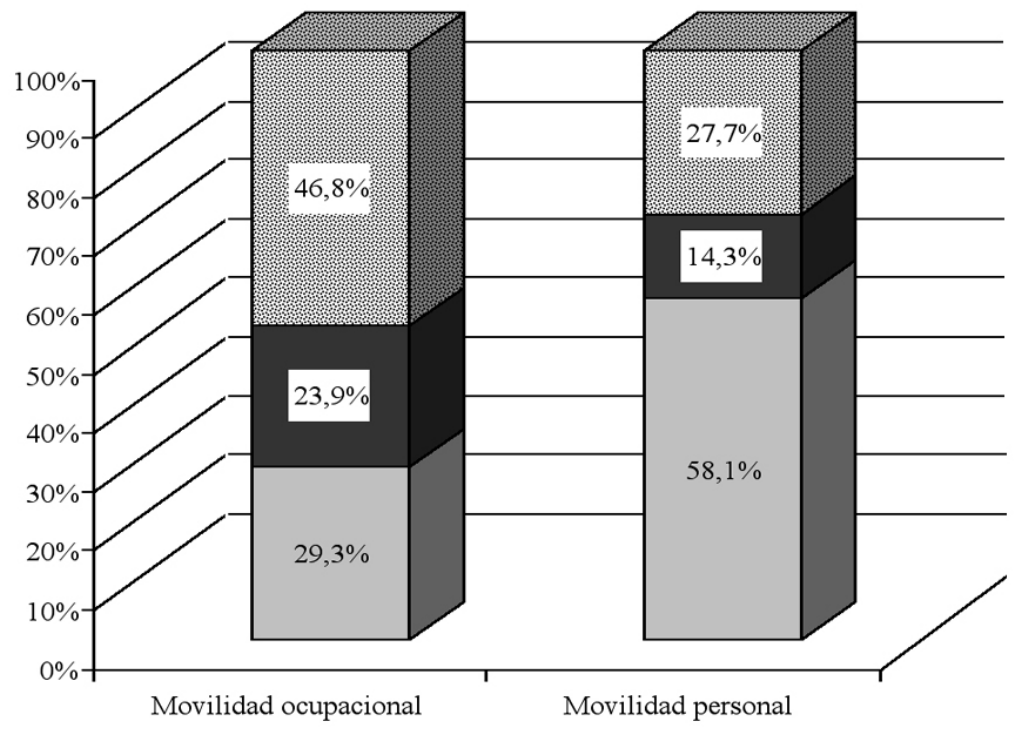

$\square$ Medios no motorizados $\mathbf{a}$ Transporte público $\mathbf{a}$ Transporte privado

Fuente: ATM y Generalitat de Catalunya, 2006

\section{USOS DEL TIEMPO Y LA MOVILIDAD COTIDIANA}

La vida cotidiana, sus actividades y sus desplazamientos se dan en dos planos distintos, el de la proximidad y el de la distancia. Y ello se manifiesta en los tiempos de desplazamiento, en cada uno de los recorridos que se realizan según los distintos motivos que los generan.

\subsection{LA VIDA COTIDIANA DE LA PROXIMIDAD O DE LA DISTANCIA}

Como se ha comentado, uno de los fenómenos que más influyen en la dispersión y la concentración urbana en términos territoriales o en la proximidad o la lejanía en términos de tiempo social es la localización de las actividades cotidianas respecto al lugar de residencia. Un indicador útil para valorar la distancia entre estos lugares es la autocontención municipal: la relación que existe entre los destinos localizados en el municipio de residencia y los que se encuentran fuera de él.

En las encuestas de movilidad, y concretamente en la EMQ06, se valoran los destinos de los desplazamientos en relación a su autocontención. De este modo se sabe que en la RMB, por todos los motivos, los desplazamientos que tienen su origen y su destino en el mismo municipio llegan al $71,3 \%$, lo que significa que un poco menos del $30 \%$ de los desplazamientos incluyen en su recorrido términos municipales distintos. 
Sin embargo, y respecto a la vida cotidiana de la proximidad y de la distancia, lo que es relevante analizar la autocontención en relación con cada uno de los motivos que generan el desplazamiento. En la Región Metropolitana de Barcelona la autocontención por motivos ocupacionales se sitúa en el $60,3 \%$ y por motivos personales alcanza el 81,6\%. En este primer nivel de desagregación, la movilidad relacionada con el trabajo y el estudio tiene mayores desplazamientos entre municipios distintos, y por lo tanto tiempos y espacios más distantes que en los motivos personales. Estos, en cambio, dibujan territorios más próximos, en la medida que tienen pocos destinos en municipios distintos al de sus orígenes.

Ahondando un poco más en estos datos, se pueden desagregar los motivos ocupacionales en trabajo y estudio, siendo el primero el que tiene una autocontención menor de todos los motivos contemplados en la Encuesta. Sólo el 51,8\% de los desplazamientos por trabajo se dan en el mismo municipio, un porcentaje que por estudios llega al $78,9 \%$. En los motivos personales, las compras alcanzan un 87,5\%, el ocio y el paseo un $85,2 \%$ y el acompañar personas el $83,2 \%$. Así pues, la autocontención de los desplazamientos indica, que por motivos sólo de trabajo las interrelaciones municipales son muy amplías, alcanzando la mitad de todos sus movimientos; sin embargo cuando se contemplan los desplazamientos por otras razones estas no superan el $20 \%$.

Esta ampliación espacial del los mercados de trabajo en la Región Metropolitana de Barcelona, observada en la evolución de la localización de los puestos de trabajo en el apartado anterior, se percibe también, cuando se analiza la Barcelona. Y aunque esta es la ciudad con una autocontención mayor en referencia al resto de municipios de la RMB, (dado su tamaño y su papel central), el lugar de trabajo en el mismo barrio de residencia (el recorrido más corto en distancia y en tiempo) ha pasado, en los últimos veinte años, de ser casi el $25 \%$ de todos los destinos, a situarse en el 2006 entorno al $15 \%$; también el porcentaje de trabajo en otros barrios de la ciudad ha disminuido un $5 \%$ en este periodo. Esta dinámica que sitúa los lugares de trabajo cada vez más alejados del lugar de residencia se traduce en un incremento del tiempo de desplazamiento al trabajo, aumentando los viajes que superan los 30 minutos de recorrido (Figura 10).

Figura 10. Tiempo de desplazamiento al trabajo (sólo ida) de la población ocupada (\%). Barcelona 1995-2006.

\begin{tabular}{|l|c|c|c|}
\hline & 1995 & 2000 & 2006 \\
\hline Menos de 15 minutos & 24,2 & 21,5 & 17,2 \\
\hline De 15 a 30 minutos & 51,1 & 50,1 & 47,5 \\
\hline Más de 30 minutos & 19,2 & 20,9 & 25,1 \\
\hline Es irregular & 5,3 & 5,6 & 8,6 \\
\hline NS/NC & 0,2 & 1,9 & 0,6 \\
\hline Total & 100 & 100 & 100 \\
\hline & & & \\
\hline Tiempo medio (en minutos) & $24: 30$ & $25: 44$ & $28: 15$ \\
\hline
\end{tabular}

Fuente: IERMB, 1990,1995, 2000; IERMB y IDESCAT, 2006

Sin embargo, esta dinámica de dispersión convive con un incremento de intensidad de uso del propio barrio. De este modo, y como indica la figura 11, los despla- 
zamientos por otras necesidades cotidianas, como compras, actividades lúdicas, etc..., se realizan en el ámbito de la proximidad. E1 89,9\% de la población se desplaza dentro del mismo barrio para la compra de alimentos envasados y este porcentaje disminuye en la medida que nos alejamos del centro. En la segunda corona metropolitana los espacios de proximidad son menores que en Barcelona, situándose en unos porcentajes por debajo del 50\% (Miralles y Cebollada, 2002).

Figura 11. Lugar donde la población compra habitualmente los productos envasados. (\%) Barcelona. 2000

\begin{tabular}{|l|c|c|c|c|}
\hline \multicolumn{1}{|c|}{ Ámbito } & Barcelona & Primera corona & Segunda corona & Total RMB \\
\hline Mismo barrio & 89,9 & 66,7 & 47,3 & 68,5 \\
\hline Centro del municipio & 0,7 & 7,9 & 13,4 & 7,2 \\
\hline Otros barrios del municipio & 4,1 & 9,4 & 13,7 & 8,9 \\
\hline Barcelona & 0,0 & 1,3 & 0,7 & 0,6 \\
\hline Ciudades maduras & 0,0 & 0,1 & 5,6 & 1,9 \\
\hline Otros municipios & 2,1 & 9,4 & 12,9 & 7,9 \\
\hline Indistintamente & 2,8 & 4,9 & 6,1 & 4,6 \\
\hline NS/NC & 0,4 & 0,3 & 0,2 & 0,3 \\
\hline Total & 100,0 & 100,0 & 100,0 & 100,0 \\
\hline
\end{tabular}

Fuente: IERMB, 2000

En Barcelona es donde la intensificación del barrio, como espacio de compras es más notoria. Así y como se ve en las figuras siguientes, el barrio como lugar de compras de los alimentos envasados se ha incrementado casi 10 puntos en los últimos veinte años, también crece como lugar donde realizar las compras del calzado y la ropa. Los destinos que más disminuyen son lo que expresan mayor distancia y tiempo de desplazamiento (Figura 12 y Figura 13). También las actividades de ocio se aproximan al barrio de residencia. En esta misma dirección se expresan los ciudadanos cuando se les pregunta donde viven las personas con las que se relacionan con más frecuencia. En Barcelona más del $70 \%$ viven en el mismo municipio y de estas más del 35\% en el mismo barrio, un porcentaje que se ha incrementado desde mediados de los años 90 . Todo esto indica alejamiento, respecto el lugar de trabajo, proximidad en el resto de actividades.

Figura 12. Lugar donde los miembros del hogar compran habitualmente los alimentos envasados (\%). Barcelona, 1985-2006.

\begin{tabular}{|l|c|c|c|c|c|}
\hline & 1985 & 1990 & 1995 & 2000 & 2006 \\
\hline Mismo barrio & 82,9 & 84,6 & 83 & 89,9 & 91,6 \\
\hline Centro de Barcelona & - & 1,2 & 0,9 & 0,7 & 1 \\
\hline Otros barrios de Barcelona* & 10,4 & 7 & 7,3 & 4,1 & 5,3 \\
\hline Otros municipios & 1,9 & 1,1 & 3,8 & 2,1 & 0,4 \\
\hline Indistintamente & 4,7 & 5,7 & 4,4 & 2,8 & 1,5 \\
\hline NS/NC & 0,1 & 0,3 & 0,6 & 0,4 & 0,2 \\
\hline Total & 100 & 100 & 100 & 100 & 100 \\
\hline
\end{tabular}

*El año 1985 esta categoría incluía la anterior

Fuente: IERMB, 1990,1995, 2000. Barcelona: IERMB y IDESCAT, 2006 
Figura 13. Lugar donde los miembros del hogar compran habitualmente la ropa y el calzado (\%). Barcelona, 1985-2006.

\begin{tabular}{|l|c|c|c|c|c|}
\hline & 1985 & 1990 & 1995 & 2000 & 2006 \\
\hline Mismo barrio & 40,9 & 43,5 & 54,2 & 50,9 & 51,5 \\
\hline Centro de Barcelona & 19,8 & 7 & 17,1 & 21,6 & 21,5 \\
\hline Otros barrios de Barcelona* & 8,1 & 17 & 12,9 & 7,6 & 13,3 \\
\hline Otros municipios & 1,2 & 1 & 0,8 & 1,1 & 0,7 \\
\hline Indistintamente & 29 & 29 & 14,3 & 18,1 & 12,2 \\
\hline NS/NC & 1,1 & 2,4 & 0,6 & 0,6 & 0,8 \\
\hline Total & 100 & 100 & 100 & 100 & 100 \\
\hline
\end{tabular}

Fuente: IERMB, 1990,1995, 2000. IERMB y IDESCAT, 2006

\subsection{EL TIEMPO DE DESPLAZAMIENTO}

El tiempo es un todo continuo, dedicado a distintas actividades. Es el tiempo de la vida cotidiana, expresado como tiempo social, en la medida que en él conviven las distintas tareas en conflicto y/o complementariedad. Dando lugar al concepto de conciliación de la vida laboral y familiar. Entre otros elementos, ello es posible gracias a la movilidad como la suma de desplazamientos que permiten llegar a los lugares donde se desarrollan las distintas actividades diarias (Miralles, 2002). Además de los motivos - $\mathrm{o}$ actividades- que nos movilizan, los medios de transporte utilizados son, también, esenciales para contextualizar el tiempo de desplazamiento, pues de ellos depende la velocidad de estos, es decir la relación entre distancia y tiempo.

Distintos análisis han evaluado el tiempo total que los barceloneses destinan diariamente a los desplazamientos, los resultados obtenidos en todos ellos son bastante similares, al considerar que este tiempo esta situado entre los 80 y los 95 minutos. Unos valores similares a los que tienen los ciudadanos de otros países europeos ${ }^{4}$, superiores en hombres y en la población más joven.

Los motivos, los medios de transporte utilizados y las características de la población influyen en los tiempos de desplazamiento. En días laborables, los desplazamientos por motivos ocupacionales tienen una duración superior media de 22,03 minutos por recorrido y los personales de 19,41 minutos. Es en Barcelona donde los desplazamientos suponen más tiempo que el resto de las coronas metropolitanas, con unos valores que en Barcelona superan los 23 minutos y en la segunda corona los 20 minutos en los motivos ocupacionales. En los personales los valores van de los 21,2 minutos en la ciudad de Barcelona a los 17,9 en la segunda corona. Si la valoración incluye sólo el trabajo el tiempo de recorrido es aún mayor situándose en casi 26 minutos en Barcelona y en la primera corona y en casi 22 en la segunda corona. En cambio, los recorridos por estudios tienen una media global de 17,6 minutos en toda la RMB.

En relación a la movilidad personal, los desplazamientos con una media más alta son los dedicados a pasear (35,02 minutos). Por el contrario, los movimientos para

4 Eurostat series Stadistics in focus, Transport. 
acompañar personas (básicamente niños a la escuela) y las compras cotidianas tienen unos valores medios de alrededor de 13 minutos. Otros motivos personales están entre los 17 y los 19 minutos.

La elección del medio de transporte condiciona la duración temporal media del trayecto. Así, de los tres modos de transporte en que estos se dividen, son los no motorizados los que presentan un valor temporal menor, con unos 15 minutos de media, los transportes privados tienen unos valores medios alrededor de los 21 minutos y los transportes públicos superan los 37 minutos. A nivel territorial el transporte que tiene más variabilidad es el público, que en la medida que nos alejamos de la ciudad de Barcelona aumenta sus valores temporales. Una cuestión relacionada con la oferta de transporte, mucho más amplia -en servicios, frecuencia e intermodalidaden el centro metropolitano, especialmente en Barcelona, que en las periferias.

Las características personales según genero, edad y situación laboral también condicionan los tiempos de la movilidad. Así se observa que los hombres hacen trayectos más largos en tiempo $\left(22,36^{\prime}\right)$ que las mujeres $\left(20,34^{\prime}\right)$. Unos tiempos que indican que ellas realizan más desplazamientos de proximidad que ellos. En lo referente a la edad se observa que los colectivos más jóvenes (de 4 a 15 años) y los mayores de 64 años presentan comportamientos opuestos, ya que los primeros son el grupo más móvil y el que utiliza menos tiempo en desplazamientos, con una media de 13 minutos y en cambio los mayores son los que sus trayectos son más largos. Las personas situadas en los tramos de edad intermedios tienen valores de tiempo de trayecto similares, alrededor de los 22 minutos por recorrido. La situación laboral es la que marca muchos de los valores de tiempo antes mencionados por la edad, al ser las personas inactivas (las menores de 16 años y las mayores de 64) aquellas que tienen valores extremos.

\section{CONCLUSIONES}

La distribución de la población, la localización de las actividades, la ocupación del territorio y los modelos de movilidad, entre otros elementos, muestran dos dinámicas metropolitanas distintas que se perciben como dos modelos de ciudad contrapuestos. Uno el de los centros urbanos, en la ciudad de Barcelona y su continuo urbano, y en algunas ciudades medias del entorno. El otro el que caracteriza los espacios más externos y periféricos de las ciudades, especialmente en la segunda corona metropolitana. Estos dos modelos influyen decididamente en la vida cotidiana de sus ciudadanos y, en especial en sus territorios y sus tiempos de la cotidianidad, expresados en espacios de proximidad o, por el contrario, de lejanía.

Se plantea, pues, la Región Metropolitana de Barcelona, como el resultado de estas dos dinámicas. Una, la ampliación de la ciudad real, del espacio urbano más allá de sus límites administrativos, como consecuencia, principalmente, del alejamiento del lugar de trabajo respecto a la residencia. Unas dinámicas que dibujan mercados de trabajo cada vez más plurimunicipales, más metropolitanos, donde los limites municipales pierden sus funciones e incluso sus formas. En estos espacios se subraya la lejanía y requieren de los transportes mecánicos, normalmente los medios privados, y son consumidores netos de tiempo de desplazamiento. 
La otra dinámica, por el contrario, es la intensificación del barrio como espacio de vida cotidiana, allí donde se vive y a la vez se compra, se utilizan los servicios y los equipamientos lúdicos y culturales. Esta acentuación del barrio implica ahorros de distancia y de tiempo. Se busca proximidad e inmediatez, con medios de transporte no mecánicos, especialmente el ir andando.

Estas dos dinámicas metropolitanas, paralelas y complementarias se dan en la Región Metropolitana de Barcelona, un espacio definido por un único mercado de trabajo, pero donde se han llevado a cabo políticas urbanas de compacticidad y de multifuncionalidad, que han revitalizado las unidades vecinales, los barrios, como espacios de vida cotidiana. Todo ello aumenta, por un lado los recorridos y los tiempos de los movimientos, por el otro, se incrementan los desplazamientos en los ámbitos más próximos, con la voluntad de minimizar los tiempos de la movilidad.

\section{BIBLIOGRAFIA}

AJUNTAMENT DE BARCELONA (2008): "Mercat de treball". Barcelona economia; Indicadors econòmics de Barcelona $i$ de la regió metropolitana, $\mathrm{n}^{\circ} 68,61-70$. http://www.bcn.es/publicacions/b_economia/be68.pdf

AUTORITAT DE TRANSPORT METROPOLITÀ (ATM) I GENERALITAT DE CATALUNYA (DEPARTAMENT DE POLITICA TERRITORIAL I OBRES PÚBLIQUES (PTOP)) (2006): Enquesta de mobilitat quotidiana 2006. Barcelona. Generalitat de Catalunya.

BAILLY, J.P. (2005): Noveaux rythmes urbains et organisation des transports. París. Conseil National des transport (CNT). Bonfiglioli, S.:" Los usos del tiempo en la ciudad.” Jornada Europea ¿Hacia dónde va nuestro tiempo? Escenarios de futuro Barcelona .Febrero. 2009.

CAMAGNI, R., GIBELLI, M.C. Y RIGAMONTI, P. (2002): "Urban mobility and urban form: the social and environmental costs of diferent patterns of urban expansion". Ecological Economics, nº40, 191-216.

CAPEL, H. (2005): La morfología de las ciudades. II aides facere: técnica, cultura y clase social en la construcción de edificios. Barcelona. Ediciones del Serbal.

CASTAÑER, M., VICENTE, J. Y BOIX, G. (eds.) (2001): Áreas urbanas y movilidad laboral en España. Girona. Universitat de Girona.

CEBOLLADA, A. (2006): “Aproximación a los procesos de exclusión social a partir de la relación entre el territorio y la movilidad cotidiana". Documents d'Anàlisi Geogràfica, no 48, 105-121.

CRANG, M.(2001): Rhythms of the city: temporalised space and motion in May, J. y Thrift (eds) Timespaces. Grographies of temporality. London. Roudge

DAVOUIDI, S.; FARINÓS DASÍ, J.; PAUL I CARRIL, V. Y DE VRIES A. (2009): $E l$ desarrollo territorial: entre la perspectiva ambiental, la cohesión social y el crecimiento económico, en Territorios, Sociedades y políticas (Feria, J.M.; García, A. y Ojeda, J.F. (eds)). Sevilla, Universidad Pablo Olavide y AGE,

DE RUS MENDOZA, G.; BETANCOR CRUZ, O.; CAMPOS MÉNDEZ, J. (2006): Evaluación Económica de proyectos de transporte. Washington D.C.. Banco interamericano de desarrollo. 
DURAN, M. A. (2007): El valor del tiempo ¿cuántas horas te faltan al día? Madrid. Espasa Calpe.

FERIA, J.M. Y SUSINO, J. (2005): Movilidad por razón de trabajo en Andalucía, 2001. Sevilla. Instituto de estadística de Andalucia.

GIDDENS, A (1984): The constitution of the society. Outline of the theory of structuralism. Berkeley. University California Press.

IERMB (1990, 1995, 2000): Enquesta de condicions de vida i hàbits de la població, Barcelona, IERMB (http://www.iermb.uab.es/)

IERMB y IDESCAT (2006): Enquesta de condicions de vida i hàbits de la població de Catalunya, Barcelona, IERMB y IDESCAT (http://www.idescat.cat/)

MANCOMUNITAT DE MUNICIPIS DE L'AREA METROPOLITANA DE BARCELONA (2003): El territori Metropolità de Barcelona. Dades bàsiques, evolució recent i perspetives. Barcelona.

MAY, J. Y THRIFT (eds) (2001): Timespaces. Grographies of temporality. London. Roudge.

MIRALLES-GUASCH, C. (2002): Transporte y ciudad. El binomio imperfecto. Barcelona. Ariel.

MIRALLES-GUASCH, C. (2006): Usos del temps y la mobilitat. Barcelona. Ajuntament de Barcelona.

MIRALLES-GUASCH, C Y CABOLLADA, A. (2002): "Mobilitat laboral, per compres i lliure", en Enquesta de la Regió de Barcelona 2000. Informe General (Giner, S. dir.) Barcelona. Mancomunitat de Municipis de l'AMB y Diputació de Barcelona.

MIRALLES-GUASCH, C. Y DONAT, C. (2007): “Analisi de l'oferta i la demanda de polígons d'activitat a Catalunya “. Papers, nº45, 8-36.

MÜCKENBERG, H. (2009): Família, política del temps i desenvolupament urbà. L'Exemple de Bremen. Barcelona. IERMB.

MUÑOZ, F. (2005): La producció residencial de baixa densitat. Elements de Debat Territorial 21. Barcelona. Diputació de Barcelona.

MUÑOZ, F. (2006): Urbanalització. La producció residencial de baixa densitat a la província de Barcelona 1985-2001. Tesis de doctorado. Bellaterra, Universitat Autònoma Barcelona.

PARKES, D. Y THIRF, N. (1980): Times, espace and places. A chronogeographic perspective. Chichester. John Wiley \& Sons.

PIRMB (2003): Transport Públic i Treball. Barcelona. Beta editorial.

pp.199-238

PUJADES RÚBIES, I. (2009): Movilidad residencial y expansión urbana en la Región Metropolitana residencial y expansión urbana en la Región Metropolitana de Barcelona, 1982-2005. Scripta Nova [En línea]. Barcelona: Universidad de Barcelona, 15 de mayo de 2009, vol XIII, $\mathrm{n}^{\circ} 290<\mathrm{http} / /$ www.ub.es/geocrit/sn/sn-290.htm> [12 de junio de 2009]. ISSN: 1138-9788.

PRATS, M. (1998) Gènere, ús del temps i geografia. Documents d'Anàlisi Geogràfica, 32, p. 175-188.

RIERA, P. (1989): Les areas funcionales a Catalunya. Bellaterra. Publicacions de la Universitat Autònoma de Barcelona.

ROBERT, R. (1992): Tempo Rubato. Como. Red edizioni. 
ROQUER SOLER, S. (2007): "Movilidad residencia-trabajo y características sociodemograficas de la población española. Un estudio de sus relaciones a través del censo de 2001". Boletin del AGE, $\mathrm{n}^{\circ}$ 4,187-214.

SALOM, J. CASADO, (2007): "Movilidad cotidiana y mercados locales de trabajo en la Comunidad Valenciana 1991-2001". Boletín de la AGE, nº 44, 5-28

SERRA, A. (2007) Vida quotidiana en un espai urbà transformat. El Mercadal de Girona des d'una perspectiva de gènere. Documents. Anàlisis Geográfica. 49, pág 161-180.

TABBONI, S. (2006): Les temps sociaux. París. Armand Colin.

TORNS, T. (2007): El tiempo de trabajo y las relaciones de género: las dificultades de un cambio ineludible en Trabajo, género y tiempo social, (Prieto, C. ed.). Madrid, Hacer editorial y Editorial complutense, pp.269-278.

WIEL, M. (1999): « Mobilité, systeme d'interactions sociales et dynamiques territoriales ». Espace, populations et societés, $\mathrm{n}^{\circ}$ 2, 187-194. 